\title{
AN UPGRADE PROPOSAL OF INJECTION BUMP SYSTEM FOR HLS*
}

\author{
Lin Wang, Weimin Li, Hongliang Xu, Shancai Zhang, Guangyao Feng, Zuping Liu,
} NSRL of USTC, P.R.China

\section{Abstract}

The current injection bump system in HLS storage ring, which is designed 8 years ago, has been operated 5 years up to the present with acceptable performance. Due to inherent limitation of HLS (Hefei Light Source) storage ring, there are some deficiencies of the design. For the potential of full energy injection, reasonable design objective was summed up. Then, physical design of new bump system was brought forward. Using simple formula, the acceptance to injected beam and perturbation on stored beam were estimated approximately. Finally, the ELEGANT software was used to simulate the injection process with new designed bump system. The simulation results showed that, the injection efficiency would be higher than $90 \%$ and perturbation on stored beam would be small enough, which are very essential to full energy injection and top-up operation of HLS.

\section{INTRODUCTION}

HLS (Hefei Light Source) was constructed in 1980's at China Mainland, and its primary parameters were briefly listed in table 1. During Phase II Project of NSRL between 1999 and 2004, some central systems at storage ring were upgraded ${ }^{[1]}$, including RF cavity and generator system, power supply of main magnets, injection system and control system. In the physical design of upgraded injection system, concentrated four-kicker local bump system was employed ${ }^{[2 \sim 4]}$, where four ferrite-core window-type kicker magnets were installed on the straight section whose length is $3 \mathrm{~m}$. Figure 1 showed the layout of upgraded bump system. Without quadrupole between kickers, the form of bump is dependent on the angle of each kicker only. When kick angle met the conditions (1), a closed local bump without slope at the injection point was produced.

$$
\theta_{1}=-\theta_{2}=-\theta_{3}=\theta_{4}=h / l,
$$

where $\boldsymbol{h}$ is height of local bump, $\boldsymbol{l}$ is the distance between kickers. Table 2 gives main parameters of this local bump system. While bump is independent of lattice parameters, there are some disadvantages, for example, as the result of large kick angle, the injection process is very sensitive to the errors of injection system and storage ring [5]; there is not possibility to implement full energy injection due to magnetic saturation in ferrite core of kickers. Simulation and operation of current injection system showed that its performance is not very comfortable. To improve the injection process of HLS and the potential of increasing the injection energy, a proposal

\footnotetext{
*Work supported by National Natural Science Foundation of China (10575096)

\#wanglin@ustc.edu.cn
}

of a new injection system was brought forward and discussed in this paper.

Table 1: Main parameters of HLS

\begin{tabular}{|l|l|}
\hline Injection/operation energy & $200 / 800 \mathrm{MeV}$ \\
\hline Circumference & $66 \mathrm{~m}$ \\
\hline Focusing type & Separate TBA \\
\hline Emittance & $\sim 160 \mathrm{~nm} \bullet \mathrm{mrad}$ \\
\hline Harmonic number & 45 \\
\hline Transverse tunes & $3.54 / 2.60$ \\
\hline Betatron coupling & $\sim 10 \%$ \\
\hline
\end{tabular}

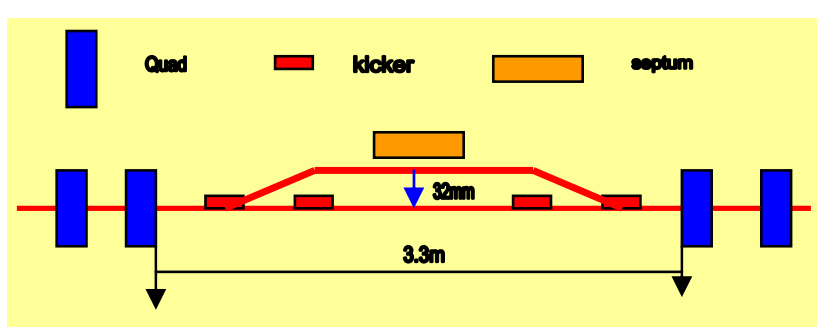

Figure 1: Layout of current bump system in HLS.

Table 2: Main parameters of current local bump system

\begin{tabular}{|l|l|}
\hline Kick angle of each kicker & $52 \mathrm{mrad}$ \\
\hline Deflecting angle of septum & $105 \mathrm{mrad}$ \\
\hline Height of local bump & $32 \mathrm{~mm}$ \\
\hline Effective length of kicker & $\sim 300 \mathrm{~mm}$ \\
\hline Magnetic field of kicker under $200 \mathrm{MeV}$ & $\sim 1160$ Gauss \\
\hline
\end{tabular}

\section{LOCAL BUMP DESIGN}

In the upgrade proposal of beam transfer line, the matching between transfer line and storage ring was perfect and the local bump height is only $26 \mathrm{~mm}$ due to moving of septum position. The simplest local bump is composed of 2 kickers, between which betatron phase advance is $\pi$, which is a strong constraint on lattice. So, bump system contained three kickers was employed before Phase II Project. The form of bump is dependent on lattice functions, moreover the bump height and slope at injection point is relevant and can't be adjusted independently. Sometimes, the bump form is not suitable to beam injection. So, the bump system consisting of four kickers was considered. Assuming the kickers are symmetric with regard to middle point of straight section, the conditions of closed local bump are as following 


$$
\begin{aligned}
& \theta_{1}=-\left(\operatorname{Csc}\left(\Delta \phi_{k 1 \rightarrow k 2}\right)\left(\begin{array}{l}
-\bar{h} \operatorname{Cos}\left(\Delta \phi_{k 2 \rightarrow \text { middle }}\right) \\
+\overline{h^{\prime}} \operatorname{Sin}\left(\Delta \phi_{k 2 \rightarrow \text { middle }}\right)
\end{array}\right)\right) / \sqrt{\beta_{1}} \\
& \theta_{2}=-\left(\operatorname{Csc}\left(\Delta \phi_{k 1 \rightarrow k 2}\right)\left(\begin{array}{l}
\bar{h} \operatorname{Cos}\left(\Delta \phi_{k 1 \rightarrow \text { middle }}\right) \\
-\overline{h^{\prime}} \operatorname{Sin}\left(\Delta \phi_{k 1 \rightarrow \text { middle }}\right)
\end{array}\right)\right) / \sqrt{\beta_{2}} \\
& \theta_{3}=-\left(\operatorname{Csc}\left(\Delta \phi_{k 1 \rightarrow k 2}\right)\left(\begin{array}{l}
\bar{h} \operatorname{Cos}\left(\Delta \phi_{k 1 \rightarrow \text { middle }}\right) \\
+\overline{h^{\prime}} \operatorname{Sin}\left(\Delta \phi_{k 1 \rightarrow \text { middle }}\right)
\end{array}\right)\right) / \sqrt{\beta_{2}} \\
& \theta_{4}=-\left(\operatorname{Csc}\left(\Delta \phi_{k 1 \rightarrow k 2}\right)\left(\begin{array}{l}
-\bar{h} \operatorname{Cos}\left(\Delta \phi_{k 2 \rightarrow \text { middle }}\right) \\
-\overline{h^{\prime}} \operatorname{Sin}\left(\Delta \phi_{k 2 \rightarrow \text { middle }}\right)
\end{array}\right)\right) / \sqrt{\beta_{1}} \\
& \bar{h}=h_{\text {middle }} / \sqrt{\beta_{\text {middle }}}, \overline{h^{\prime}}=\sqrt{\beta_{\text {middle }}} h_{\text {middle }}^{\prime}
\end{aligned}
$$

where $\boldsymbol{h}_{\text {middle }}$ is height of bump at the middle point, $\boldsymbol{h}_{\text {middle }}^{\prime}$ is the slope. The height and slope can be varied by adjusting matching between kickers. The kick angle is dependent on the requirement of bump height, also dependent on the betatron function and betatron phase advance at kickers.

To reduce the requirement on kicker strength, the distributed local bump system was adopted, where four kickers were installed at arcs and straight sections, as showed in figure 2. The requirements on kickers were listed in table 3 . The kick angles of first and last kickers at arcs, where beta function is small, are relatively large. The kick angles of second and third kickers are very small.

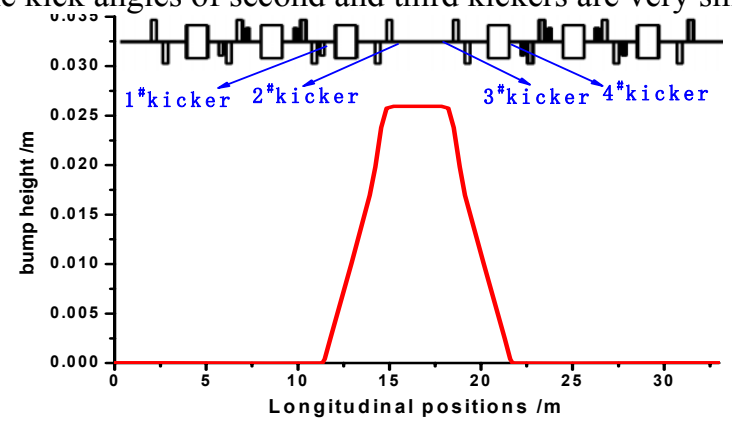

Figure 2: Sketch map of new designed local bump.

Table 3: Main parameters of new designed bump system

\begin{tabular}{|l|l|}
\hline Kick angle of first and fourth kickers & $6.86 \mathrm{mrad}$ \\
\hline Kick angle of second and third kickers & $-0.50 \mathrm{mrad}$ \\
\hline Deflecting angle of septum & $105 \mathrm{mrad}$ \\
\hline Height of local bump & $26 \mathrm{~mm}$ \\
\hline Effective length of kicker & $\sim 100 / 30 \mathrm{~mm}$ \\
\hline Magnetic field of kicker under $200 \mathrm{MeV}$ & $\sim 458 / 111 \mathrm{Gauss}$ \\
\hline
\end{tabular}

\section{WAVEFORM CHOISE}

Next, the parameters of pulsed magnetic field waveform should be determined. The injection efficiency is dependent on the properties of injected beam and waveform parameters of kicker. The emittance and energy spread of injected beam is determined by linac. The horizontal and vertical beam position jitters are relevant to the quality of hardware in linac and transfer line and these parameters were estimated by numerical

02 Synchrotron Light Sources and FELs simulation of transfer line ${ }^{[6]}$. The main parameters of injected beam are listed in table 4.

Table 4: Main parameters of injected beam

\begin{tabular}{|l|l|}
\hline Beam emittance & $0.5 \mathrm{~mm} . \mathrm{mrad}$ \\
\hline Pulse length & $0.2 \mu \mathrm{s}$ \\
\hline Position jitter & $< \pm 1 \mathrm{~mm}$ \\
\hline Slope jitter & $< \pm 1 \mathrm{mrad}$ \\
\hline Twiss parameters & Matched to storage ring \\
\hline Energy spread & $0.5 \%$ \\
\hline Ideal horizontal position & $39 \mathrm{~mm}$ \\
\hline Ideal horizontal slope & $0 \mathrm{mrad}$ \\
\hline
\end{tabular}

To obtain high injection efficiency and stable performance of system, the injection acceptance should be large enough. The parameters of waveform can be determined according to following equation,

$$
h_{0} f\left(t_{i n j}+n \cdot T_{0}\right)+A(n)<h_{s e p},
$$

where $\boldsymbol{h}_{\mathbf{0}}$ is maximum bump height, $\boldsymbol{h}_{\text {sep }}$ is position of septum plate, the $\boldsymbol{A}(\boldsymbol{n})$ is betatron oscillation amplitude at nth turn, The $\boldsymbol{f}[\boldsymbol{z}]$ is mathematical description of waveform. The perturbation from nonlinear field was ignored. For HLS, the horizontal betatron tune is 3.54, and the distance of injected beam to septum plate at second turn is smallest, which is used as acceptance limitation in calculation. To simplify calculation, the waveform of kicker was assumed as half sine form, in which the width of pulse or frequency of sine wave is the only variable. Assuming injection was started at the peak of half sine form, so it is direct to calculate the needed pulse width, which is less than $1.0 \mu$ s. When injection was started at dropping side of sine form, the shrinking of local bump is quicker. Choosing the better injection time, the requirement on kick pulse width would be loosed. The kick pulse width was selected as $1.32 \mu$ s and calculation showed that the injection acceptance is enough large.

The errors of injection system would lead to leakage of local bump. It maybe be reduce the injection efficiency, at same time, it would perturb the stored beam and results in centre-mass emittance growth even in stored beam loss. Considering linear effect, the centre-mass emittance growth can be expressed as following,

$\varepsilon=\left(\bar{x}^{2}+{\overline{p_{x}}}^{2}\right) / 2$

$\bar{x}(n)=\sum_{j}^{m} \sum_{i=1}^{n} f\left[i \cdot T_{0}\right] \operatorname{Sin}\left[(n-i) \mu+\phi_{j}\right]$

$\overline{p_{x}}(n)=\sum_{j}^{m} \sum_{i=1}^{n} f\left[i \cdot T_{0}\right] \operatorname{Cos}\left[(n-i) \mu+\phi_{j}\right]$

where $\boldsymbol{i}$ and $\boldsymbol{j}$ are indices for turns and kicker numbers.Usually, the errors of injection system can be expressed by following approximate formula, which 
includes the time jitters and amplitude jitters of kickers, inhomogeneity of magnetic field, and the waveform difference between different kickers.

$$
f(t)=\sqrt{\beta} \theta\left(1+\delta_{1}\right) \operatorname{Sin}\left(2 \pi\left(1+\delta_{2}\right) f\left(t+\delta_{3}\right)\right)\left(1-\lambda \cdot h^{2}(t)\right),
$$

where $\lambda$ is sextupole coefficient coming from field ununiformity, $\boldsymbol{\delta}_{\boldsymbol{i}}$ are random number and their ranges are given in table 5 . The perturbation on stored beam can be estimated approximately by above simple expressions. The statistical calculation results showed that, the perturbation on stored beam is very small and the emittance growth is less than 10 and $0.5 \mathrm{~nm} \bullet \mathrm{rad}$ in horizontal and vertical plane respectively. Compared with the beam emittance, this perturbation is ignored almost.

Table 5: Errors of injection system

\begin{tabular}{|l|l|}
\hline Amplitude jitter & $< \pm 0.5 \%$ \\
\hline Time jitter & $< \pm 5 \mathrm{~ns}$ \\
\hline Waveform difference & $<0.3 \%$ \\
\hline Uniformity of field & $<1 \%$ at $32 \mathrm{~mm}$ \\
\hline Rotation angle & $< \pm 1 \mathrm{mrad}$ \\
\hline Leak field of septum & $<0.3 \%$ at $26 \mathrm{~mm}$ \\
\hline
\end{tabular}

As a conclusion of choice of waveform parameters, the pulse width of magnetic field is narrower than that of current system and results in quicker shrinkage of local bump. This is beneficial to high injection efficiency. At same time, the weak effective perturbations on stored beam $\sqrt{\boldsymbol{\beta}_{\boldsymbol{i}} \cdot \boldsymbol{\delta} \boldsymbol{\theta}_{\boldsymbol{i}}}$ of each kicker are essential to avoid the loss of stored beam and to assure orbit stability.

\section{NUMERICAL SIMULATION}

Above estimations of injection efficiency and perturbation on stored beam are approximate. To check up the performance of injection system, the software ELEGANT ${ }^{[7]}$ was used to simulate the dynamics of injected beam and stored beam. In numerical simulation, the injected and stored beam was composed of 10000 macro-particles, whose parameters of transverse and longitudinal distribution are given in table 1 and table 2 . Then, the errors of injection system, the nonlinear fields, and the focusing errors of lattice are included in simulation. The alignment errors of main magnets in storage ring were not considered in simulation. This case is similar to that closed orbit distortion was eliminated by orbit correction system. To obtain more believable conclusion, 1000 times simulation were made using random errors. Using numerical simulation results, the injection efficiency and perturbation on stored beam were extracted statistically. The numerical simulation results are similar to that of approximate estimation. The statistical result of injection efficiency is about $94 \%$. With the improvement of injected beam properties, the injection efficiency would be higher. Figure 3 and figure 4 give the horizontal and vertical trajectories of stored beam during injection process. The maximum horizontal and vertical centre-mass emittance growth of stored beam is less than 20 percent of beam emittance.

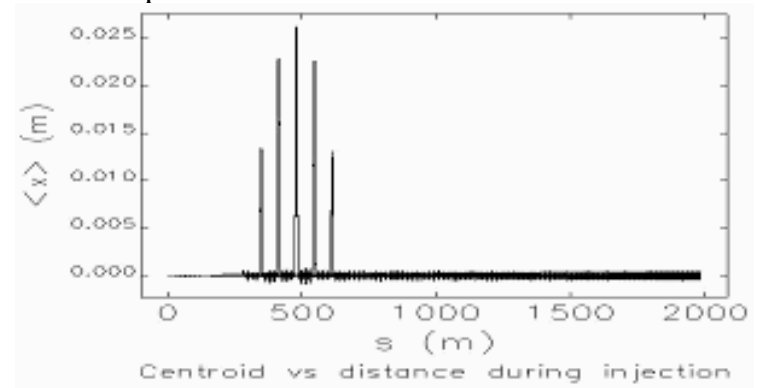

Figure 3: Horizontal orbit perturbation of stored beam.

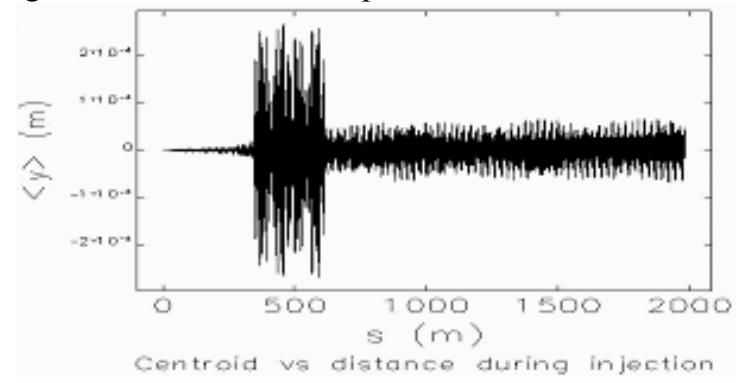

Figure 4: Vertical orbit perturbation of stored beam.

\section{CONCLUSION}

With new designed injection system, the injection efficiency is very high with current linac of HLS, and the perturbation on stored beam is ignorable. At same time, the used magnetic field of kickers is low, which may be enhanced 4 times and used in full energy injection of HLS in the future upgrade. And then, there exists potential of top-up operation due to small perturbation on stored beam and a little loss of injected beam.

\section{REFERENCES}

[1] Zuping Liu, Xinyi Zhang, "NSRL Phase II Project: A Brief Introduction and Status", Journal of Synchrotron Radiation, 1998.

[2] L.Shang, X.Q.Wang, Y.J.Pei, "Tracking study of the new injection bump system of the HLS ring", Nucl. Instrum. Methods Phys. Res. A 406(1998) 177-181.

[3] X.Q.Wang, Y.J.Pei, L.Shang, et al, "The Progress of Development of Four Kickers Bump System for Injection in HLS Ring", Proceedings of PAC'99(1315-1317).

[4] K.J.Fan, X.Q.Wang, Y.J.Pei, “A proposed injection system for HLS”, Nucl. Instrum. Methods Phys. Res. A 450(2000) 573-578.

[5] Zuping Liu, Weimin $\mathrm{Li}$, Hongliang $\mathrm{Xu}$, et al, "Analysis of some phenomena in the commissioning of the storage ring in Phase II Project of the HLS". HEP\&NP (in Chinese), Vol.30, p72-74(2006).

[6] L Wang, "The beam dynamics study of Hefei Light Source storage ring" (in Chinese), PhD, 2006.

[7] M.Borland, "ELEGANT: A Flexible SDDSCompliant Code for Accelerator Simulation", Advanced Photon Source LS-287, September 2000, USA. 